\title{
Galaxy morphology and evolution from SWAN adaptive optics imaging ${ }^{\star}$
}

\author{
G. Cresci $^{1}$, R. I. Davies ${ }^{2}$, A. J. Baker ${ }^{3,4}$, F. Mannucci ${ }^{5}$, M. D. Lehnert ${ }^{2}$, T. Totani ${ }^{6}$, and Y. Minowa ${ }^{7}$ \\ 1 INAF - Osservatorio Astrofisico di Arcetri, Largo E. Fermi 5, 50125 Firenze, Italy \\ e-mail: gcresci@arcetri.astro.it \\ 2 Max-Planck-Institut für extraterrestrische Physik, Postfach 1312, 85741 Garching, Germany \\ 3 Jansky Fellow, National Radio Astronomy Observatory \\ 4 Department of Astronomy, University of Maryland, College Park, MD 20742-2421, USA \\ 5 CNR-Istituto di Radioastronomia, Largo E. Fermi 5, 50125 Firenze, Italy \\ ${ }^{6}$ Department of Astronomy, Kyoto University, Kitashirakawa, Kyoto 606-8502, Japan \\ 7 Institute of Astronomy, School of Science, University of Tokyo, 2-21-1 Osawa, Mitaka, Tokyo 181-0015, Japan \\ Received 26 April 2006 / Accepted 7 July 2006
}

\section{ABSTRACT}

\begin{abstract}
We present the results from adaptive optics (AO) assisted imaging in the $K_{\mathrm{s}}$ band of an area of $15 \operatorname{arcmin}^{2}$ for SWAN (Survey of a Wide Area with NACO). We derive the high resolution near-IR morphology of $\sim 400$ galaxies up to $K_{\mathrm{s}} \sim 23.5$ in the first 21 SWAN fields around bright guide stars, carefully taking into account the survey selection effects and using an accurate treatment of the anisoplanatic AO PSF. The detected galaxies are sorted into two morphological classes according to their Sérsic index. The extracted morphological properties and number counts of the galaxies are compared with the predictions of different galaxy formation and evolution models, both for the whole galaxy population and separately for late-type and early-type galaxies. This is one of the first times such a comparison has been done in the near-IR, as AO observations and accurate PSF modeling are needed to obtain reliable morphological classification of faint field galaxies at these wavelengths. For early-type galaxies we find that a pure luminosity evolution model, without evidence for relevant number and size evolution, better reproduces the observed properties of our $K_{\mathrm{s}}$-selected sample than current semi-analytic models based on the hierarchical picture of galaxy formation. In particular, we find that the observed flattening of elliptical galaxy counts at $K_{\mathrm{s}} \sim 20$ is quantitatively in good agreement with the prediction of the pure luminosity evolution model that was calculated prior to the observation. For late-type galaxies, while both models are able to reproduce the number counts, we find some hints of a possible size growth. These results demonstrate the unique power of AO observations to derive high resolution details of faint galaxies' morphology in the near-IR and drive studies of galaxy evolution.
\end{abstract}

Key words. Galaxy: fundamental parameters - galaxies: statistics - infrared: galaxies - instrumentation: adaptive optics

\section{Introduction}

One of the main objectives of modern astrophysics is understanding the process of galaxy formation and evolution. The best way to tackle this issue is studying the properties of galaxies observed at the epoch of their formation and early evolution, such as their stellar population, history of mass assembly, morphology, metallicity and interplay with the intergalactic medium. However, disentangling these processes in nearby systems is already extremely difficult, and the challenge is even greater at higher redshift, where sources are compact in size $\left(\sim 0.1^{\prime \prime}-0.3^{\prime \prime}\right)$ and larger galaxies are rare (e.g., Bouwens et al. 2004). To resolve and study the details of high-redshift galaxies using ground based telescopes, which can provide larger samples and deeper observations than space-based observations, it is necessary to overcome the blurring effects of the atmosphere through the use of adaptive optics (AO) systems. These can allow ground-based telescopes to operate at or near the diffraction limit in the nearinfrared ( $0.07 "$ in $K$ band for an $8 \mathrm{~m}$ telescope), resulting in a high angular resolution and a low background in each pixel.

Besides the technical advantages afforded by AO, nearinfrared surveys provide one of the best opportunities to

* Based on observations collected at the European Southern Observatory, Chile under programs 70.B-0649, 71.A-0482 and 073.A-0603. investigate the cosmic evolution of galaxies and their mass assembly. In particular, $K$-band $(2.2 \mu \mathrm{m})$ selected samples are ideally suited for addressing the problems of galaxy formation and evolution. First, since the rest frame near-IR luminosity is a good tracer of the galaxy stellar mass (e.g., Brinchmann \& Ellis 2000; Bell \& de Jong 2001; Mannucci et al. 2005), $K$-band surveys allow us to select galaxies according to their mass up to $z \sim 1.5\left(\lambda_{\text {rest }} \sim 0.9-1.0 \mu \mathrm{m}\right)$, rather than suffer strong biases towards star-forming and peculiar galaxies like optical surveys (e.g. Drory et al. 2004; Fontana et al. 2004). Another strong argument for selecting galaxies in the near infrared is that, due to the similarity of the spectral shapes of different galaxy types and stellar population ages in the rest frame near-IR over a wide redshift range (e.g., Mannucci et al. 2001), the selection of galaxies in the $K$ band is not affected by strong $k$-correction effects (e.g., Cowie et al. 1994). In contrast, selection in the $I$ band becomes very type sensitive beyond $z=1$, and the situation is even more extreme in the $B$ band, where the fading of early-type galaxies is substantial even at modest redshifts. Thus, near-IR samples do not depend as strongly on galaxy type as optically selected ones, which are more sensitive to recent and ongoing star formation activity (as they sample the rest-frame UV light) and are biased against old and passive or weakly star-forming galaxies.

Finally, near-IR surveys are less affected by dust extinction than optical ones, making it possible to select highly 
extinguished star-forming galaxies. The observation of the obscured dusty star formation rate is crucial for measuring the global star formation history. Calculations based on the observed rest frame UV flux (e.g., Madau et al. 1996; Connolly et al. 1997) might be significantly underestimated if a large fraction of the overall star formation at high redshift takes place in highly obscured starburst galaxies (e.g., Steidel et al. 1999; Blain et al. 2002).

Morphology is one of the most appropiate ways to characterize the properties of galaxies, and we will only reach a complete understanding of galaxies by deriving the mechanisms responsible for their morphologies. In this context, the study of galaxy size, and of the evolution of other galaxy properties according to morphological type, have made use mainly of the classification derived from deep optical HST imaging (e.g., Simard et al. 1999; Labbé et al. 2003; Trujillo \& Aguerri 2004; Pannella et al. 2006), due to the higher angular resolution achievable at optical wavelengths with HST. However, near-infrared morphology is a better tracer of the underlying mass distribution, as it is not biased towards recent star formation and is less affected by dust obscuration. By using adaptive optics, it is now possible to push the analysis of source properties (surface density, magnitude, color, morphology, etc.) as a function of source size in the near-IR to an entirely new regime, and study sources that are both faint and compact. Ample evidence already indicates that such source populations do exist - e.g., a large fraction of the $H_{A B}<21$ sources detected by Yan et al. (1998) are still unresolved at the $\sim 0.35^{\prime \prime}$ resolution provided by HST/NICMOS in the near-IR. The AO-corrected, diffraction-limited, near-IR PSF of an $8 \mathrm{~m}$ telescope is a powerful tool to study this kind of object, since the angular resolution it yields is even higher than can be obtained by HST at this wavelength.

Although the advantages of near-IR AO observations for studying how galaxies form and evolve in the early universe are clear, until now there have been only a few attempts using natural guide stars (NGS; see e.g., Larkin et al. 2000; Glassman et al. 2002; Steinbring et al. 2004; Minowa et al. 2005), due to the very small number of known extragalactic sources lying at distances $\Delta \theta \lesssim 30^{\prime \prime}$ from bright $(V \lesssim 13$ ) stars needed to correct the wavefront for AO guiding, and to the problems arising from the anisoplanaticism of the PSF in AO observations. The prospects for AO cosmology will undoubtedly improve with the widespread adoption of laser guide star (LGS) systems, since these impose less stringent requirements on the brightness of stars used for tip-tilt correction (e.g., Melbourne et al. 2005). However, to overcome the present shortage of targets for AO cosmology, it is necessary to identify and characterize extragalactic sources in the vicinity of bright guide stars (see e.g., Larkin et al. 1999; Davies et al. 2001; Christopher \& Smail 2006).

We therefore undertook a campaign of seeing-limited nearIR imaging of fields selected around stars bright enough for AO guiding $(10.3 \leq R \leq 12.4)$, blue $(B-R \leq 1.1$, in order to maximize the amount of light on the wavefront sensor), lying at high galactic latitude $(|b| \geq 15 \mathrm{deg}$, to minimize extinction and contamination by foreground stars), and with a declination suitable for observations with the ESO Very Large Telescope at low air mass $(-44 \mathrm{deg} \leq \delta \leq-13 \mathrm{deg})$. A total of 42 southern bright star fields (SBSFs) were selected and observed at seeing-limited resolution in $K_{\mathrm{s}}$ band with SOFI at the ESO New Technology Telescope. More details about the target selection and data can be found in Baker et al. (2003). The same fields have been followed up at optical wavelengths (Davies et al. 2006), and are now targets for VIMOS integral field optical spectroscopy at the ESO Very Large Telescope (VLT).
In this paper we present the results of our $K_{\mathrm{s}}$-band $\mathrm{AO}$ imaging survey of the first 21 fields in the framework of SWAN (Survey of a Wide Area with NACO), which is the AO-assisted result of these seeing-limited preliminaries. The survey will be introduced in the following section, and the observations will be briefly described in Sect. 3. The data reduction approach will be presented in Sect. 4, while the detection criteria and technique will be discussed in Sect. 5. The extraction of the morphological parameters of the detected galaxies is analyzed in Sect. 6, and the method used to distinguish between stars and galaxies is described in Sect. 7. In Sect. 8 we take into account the selection effects present in our data, discuss the completeness of the survey, and show the corrected number counts. The number counts and size-magnitude relation of the full sample of galaxies and for late and early-type systems separately are compared with the predictions of two different galaxy evolution models in Sect. 9; our conclusions follow in Sect. 10. All the magnitudes are Vega relative unless otherwise specified.

\section{The survey of a wide area with NACO}

Having already characterized large samples of objects in bright star fields, as described in the previous section, we targeted them with NACO on the VLT in order to exploit the present generation of AO technology for galaxy evolution studies. NACO comprises the NAOS Shack-Hartmann AO module (Rousset et al. 2003) mated with CONICA near-infrared camera (Lenzen et al. 1998). Our choice of NACO observing mode was dictated by our desire to complement previous HST/NICMOS surveys. First, we chose to image in $K_{\mathrm{s}}$, where NICMOS is less sensitive than in $J$ and $H$, thus making SWAN preferentially sensitive to red objects. Second, we chose to prioritize survey area over depth, in order to optimize the study of the galaxies over the last half of the Hubble time and improve SWAN's sensitivity to rare objects and its robustness against cosmic variance (the latter already enhanced by the survey's peculiarity of patching together small fields at different locations on the sky). Use of NACO's $0.054^{\prime \prime}$ pixel scale (to maximize the field of view) and the Strehl ratios of 30-60\% typically achieved in $K_{\mathrm{S}}$ result in images that are slightly undersampled. As the AO PSF is quickly changing both in time and position on the frame, in order to extract full information from our wide-field observations we have developed a new approach to account for the anisoplanatic PSF. The method was presented in Cresci et al. (2005), hereafter Paper I, along with some examples of galaxy morphology fitting using the derived model PSF.

Each NACO pointing provides a usable $\sim 0.75 \operatorname{arcmin}^{2}$ of the full $55.5^{\prime \prime} \times 55.5^{\prime \prime}$ detector area, due to losses from dithering and the central star (see, e.g., Fig. 1). Nevertheless, the anticipated survey area that will result from assembling 42 such images will be - at $\sim 30 \operatorname{arcmin}^{2}-$ some six times larger than the NICMOS survey of the HDF and flanking fields in $J$ and $H$ (Dickinson 1999; Dickinson et al. 2000). SWAN aims to combine the high angular resolution of a space-based survey with the shallower depth and wider area of a ground-based survey, thereby probing sources that are compact, faint, red, and rare more effectively than any other survey to date.

\section{Observations}

The first 21 SWAN fields were observed in $K_{\mathrm{s}}$ band with the NACO AO system at the VLT, using the visible Wave Front Sensor (WFS). An example of a SWAN image is given in Fig. 1. 


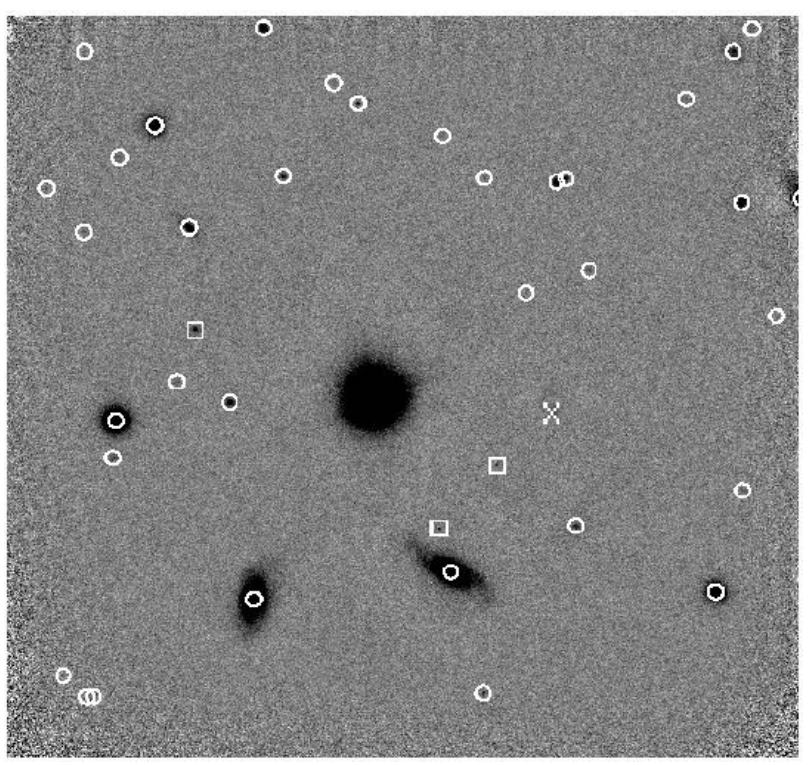

Fig. 1. Example of a $55^{\prime \prime} \times 55^{\prime \prime}$ SWAN field: SBSF 24 . The bright source in the center is the guide star, and the circles are the extended objects detected by SExtractor (SExtractor stellarity index SSI < 0.9); the squares are point sources ( $\mathrm{SSI} \geq 0.9$ ). A ghost of the bright guide star is marked with a cross. North is up and east is right.

Table 1 summarizes other observational parameters and the AO system performance during the observations. The SBSF name is given in Col. (1), and the coordinates of the guide star in the center of each field are given in (2) and (3), accurate to $\pm 0.2^{\prime \prime}$. Column (4) reports the date(s) on which each field was observed. The total integration time on each field is given in (5), and the noise measured in the resulting coadded image rescaled to $1 \mathrm{sec}$ integration time in (6). The mean airmass is reported in (7). The Strehl ratio, estimated from a series of short exposures through a narrow band filter taken before and/or after the science exposures in order to monitor the on-axis PSF, is given in Col. (8). This is calculated from the ratio of the maximum pixel to the total flux, and includes a correction for the offset of the PSF's centroid from the center of a pixel; this can be considerable (typically adding $5-10 \%$ to the Strehl ratio for the data here) due to the large $0.054^{\prime \prime}$ size of the pixels. The number of bright point sources in each field used to evaluate the isoplanatic angle at $2.2 \mu \mathrm{m}$, fitting the variation of their Strehl ratio in our $K_{\mathrm{s}}$ images (see Sect. 6), is reported in (9), and the resulting isoplanatic angle in Col. (10).

\section{Data reduction}

The data obtained were reduced using PC-IRAF (version 2.11.3) together with some scripts in IDL (version 6.0). The presence of a bright star in the center of a field less than $1^{\prime}$ across made the data reduction more complex than usual, requiring extra steps to compensate. An initial estimate of the sky background was made from the target frames after masking all bright objects in the fields. Each target frame then had the sky subtracted, was flat fielded, had any residual constant background removed, and hot pixels corrected. A mask that included dead pixels and bad regions was then applied to each frame. In order to correct for oversubtraction from very extended faint scattering (and/or emission) around bright objects, a surface was fit to each frame (ignoring regions in the object mask) and subtracted. The frames were then aligned with sub-pixel accuracy using up to several conspicuous isolated objects in the field, and averaged after rejecting high and low pixels at each point according to an estimated variance. This initial combined frame was used to generate a new object mask, and the entire data reduction process was repeated, yielding a new combined frame with much less over-subtraction. In a final step, the objects were once more masked out and a surface fitted to the background, and this was subtracted to produce the final image.

The sky is estimated by dithering, i.e., slightly moving the telescope between different frames so that different pixels sample different parts of the sky. In SWAN the offsets were chosen semi-randomly within a $7^{\prime \prime}$ box, due to the limited NACO field of view. Therefore, even if great care is used to produce the sky frames, the sky around objects larger than $\sim 3^{\prime \prime}$, i.e., for the very bright guide star and for galaxies with effective radius $R_{\mathrm{e}} \gtrsim 1^{\prime \prime}$, may be overestimated, producing a self-subtraction of some galaxy flux. This effect can produce fainter magnitudes and smaller dimensions for such bright and large objects, although these constitute less than $2 \%$ of the total sample in our fields. However, in Sect. 9 we will see that this effect introduces some systematic uncertainties in the size-magnitude relation for large galaxies.

\section{Source detection}

In each reduced SWAN field, sources were detected using SExtractor (Bertin \& Arnouts 1996), with the appropriate parameters optimized for compact sources, set to provide a positive detection for objects brighter than $1.5 \sigma$ per pixel over an area of more than 3 pixels. To improve the detection of faint sources we used a Gaussian filter ( $\sigma=1.5$ pixels) to smooth the image. False detections at the noisy borders of the mosaic and on the spikes and the ghost of the bright guide star were removed. For the former, a mask that indicated the fraction of the total integration time spent on each pixel was used; objects detected in pixels below a specified threshold were rejected. For the latter, appropriate object masks were created. Our algorithm deliberately does not push the detection to the faintest possible limit, as we are more interested in the high resolution AO morphologies of the brighter sources than in the deepest possible number counts. For this reason, our counts (see Sect. 8) are not significantly contaminated by spurious detections due to noise. The total coverage above the detection thresholds of the 21 fields is $15.3 \operatorname{arcmin}^{2}$, within which a total of 495 sources are detected down to a magnitude of $K_{\mathrm{S}} \sim 23.5\left(K_{\mathrm{AB}} \sim 25.3\right.$, see Sect. 8).

\section{Morphological fitting}

The morphological parameters of the detected galaxies were derived using GALFIT (Peng et al. 2002), a widely used software package that fits a two-dimensional image of a galaxy and/or a point source with one or more analytic functions that have been convolved with a model of the PSF. To fit the galaxies in our SWAN fields we used a single Sérsic (1968) profile,

$I(R)=I\left(R_{\mathrm{e}}\right) \times \exp \left(-b_{n} \times\left[\left(R / R_{\mathrm{e}}\right)^{1 / n}-1\right]\right)$

where $R_{\mathrm{e}}$ is the effective radius that encloses half of the light, $n$ is the Sérsic index and $b_{n}$ is a constant that varies with $n$, chosen so that $R_{\mathrm{e}}$ corresponds to the half-light radius.

GALFIT needs as an input a PSF to convolve the Sérsic profile model. We used the off-axis AO PSF model presented in Paper I, which is optimized for wide-field and high Galactic latitude observations. The off-axis PSF is determined by convolving 
Table 1. Observational parameters and AO performances for NACO observations of SWAN fields. See the text for a full description of the entries. ${ }^{a}$ CONICA was fitted with a new detector in June $2004 .{ }^{b}$ The noise is that measured in the resulting co-added image, scaled to a 1 sec integration. Its statistical properties closely follow a Gaussian distribution with additional weak wings. " ${ }^{c} \theta_{0}$ " refers to the isoplanatic angle in $K_{\mathrm{s}}$ band as measured fitting the variation of the Strehl ratio of the point sources in the fields as described in the text.

\begin{tabular}{|c|c|c|c|c|c|c|c|c|c|}
\hline $\begin{array}{c}\text { Name } \\
\text { (1) }\end{array}$ & $\begin{array}{c}\text { RA } \\
(\mathrm{J} 2000.0) \\
(2)\end{array}$ & $\begin{array}{c}\text { Dec } \\
(\mathrm{J} 2000.0) \\
(3)\end{array}$ & $\begin{array}{l}\text { Obs. } \\
\text { date }^{a} \\
(4)\end{array}$ & $\begin{array}{c}\Delta t \\
(\min ) \\
(5)\end{array}$ & $\begin{array}{c}\sigma_{\text {image }^{b}} \\
\left(\mathrm{e}^{-} \mathrm{s}^{-1}\right) \\
(6)\end{array}$ & $\begin{array}{c}\text { Mean } \\
\text { airmass } \\
(7)\end{array}$ & $\begin{array}{c}\text { Strehl } \\
\% \\
(8)\end{array}$ & $\begin{array}{l}\text { N. of } \\
\text { stars } \\
(9)\end{array}$ & $\begin{array}{c}\theta_{0}^{c} \\
\left({ }^{\prime \prime}\right) \\
(10)\end{array}$ \\
\hline SBSF 02 & 004431.88 & -295230.3 & 07.09 .04 & 54 & 0.29 & 1.08 & - & 2 & 5.90 \\
\hline SBSF 03 & 004520.62 & -295646.0 & 08.09 .04 & 54 & 0.29 & 1.24 & - & 2 & 11.20 \\
\hline SBSF 04 & 004528.05 & -293140.1 & 09.12 .04 & 68 & 0.28 & 1.22 & 33 & 2 & 10.65 \\
\hline SBSF 06 & 005034.70 & -292632.0 & 09.09.04 & 54 & 0.33 & 1.17 & 24 & 0 & [12.70] \\
\hline SBSF 08 & 005218.88 & -292717.8 & 12.09 .04 & 56 & 0.32 & 1.09 & 42 & 1 & 12.00 \\
\hline SBSF 14 & 060706.34 & -131337.1 & 15.12 .02 & 60 & 0.22 & 1.20 & - & 4 & 11.95 \\
\hline SBSF 15 & 084400.22 & -163401.1 & 17.12 .02 & 60 & 0.25 & 1.12 & 46 & 10 & 21.78 \\
\hline SBSF 16 & 091452.77 & -192617.0 & 18.12 .02 & 50 & 0.25 & 1.07 & - & 1 & 12.84 \\
\hline SBSF 17 & 094744.79 & -213712.7 & 20.03 .03 & 44 & 0.28 & 1.05 & 35 & 2 & 10.61 \\
\hline SBSF 18 & 094946.99 & -214513.3 & 17.12 .02 & 40 & 0.30 & 1.06 & - & 7 & 21.18 \\
\hline SBSF 24 & 104026.20 & -300036.5 & 21.03 .03 & 60 & 0.24 & 1.02 & 30 & 8 & 11.77 \\
\hline SBSF 27 & 125537.48 & -314241.3 & 11.04 .04 & 44 & 0.30 & 1.07 & 35 & 12 & 17.85 \\
\hline SBSF 28 & 125614.14 & -420910.9 & 11.04 .04 & 44 & 0.27 & 1.26 & 42 & 2 & 9.94 \\
\hline SBSF 34 & 134625.24 & -314547.5 & 11.04 .04 & 44 & 0.28 & 1.36 & 28 & 2 & 4.89 \\
\hline SBSF 36 & 221436.74 & -282531.6 & 05.09 .03 & 60 & 0.23 & 1.09 & 35 & 2 & 13.25 \\
\hline SBSF 37 & 224304.43 & -394929.3 & 06.09 .03 & 60 & 0.23 & 1.10 & 34 & 0 & [12.70] \\
\hline SBSF 38 & 224706.77 & -401001.3 & 05.09 .03 & 60 & 0.22 & 1.04 & 31 & 2 & 10.68 \\
\hline SBSF 39 & 224934.23 & -393305.3 & 15.06 .03 & 60 & 0.24 & 1.04 & 22 & 1 & 10.00 \\
\hline SBSF 40 & 224949.32 & -395315.0 & 12.06 .03 & 48 & 0.34 & 1.04 & 10 & 0 & 12.70 \\
\hline SBSF 41 & 225021.28 & -400738.6 & 14.06 .03 & 60 & 0.26 & 1.04 & 33 & 3 & 19.72 \\
\hline SBSF 42 & 232955.77 & -183554.1 & 16.06 .03 & 60 & 0.24 & 1.03 & 35 & 0 & [12.70] \\
\hline
\end{tabular}

the on-axis PSF in each of the fields with an elliptical Gaussian kernel elongated towards the guide star. The FWHM of the kernel depends on the distance from the guide star and on the isoplanatic angle of the field. We therefore derived the isoplanatic angle for each field fitting the variation of the Strehl ratio of the point sources across the field as described in Paper I. The obtained isoplanatic angle along with the number of point sources used in the fit are reported in Table 1. The derived isoplanatic angles for the 21 fields range from $4.9^{\prime \prime}$ to $21.8^{\prime \prime}$. In four of the fields no bright point source was available except the guide star, and therefore the average isoplanatic angle for the other fields $\left(12.7^{\prime \prime}\right)$ was assumed.

Initial guesses for GALFIT model parameters were obtained from the SExtractor source catalogs. Lacking an estimate of the Sérsic index $n$ in the SExtractor catalogs, we used $n=2$ for all the galaxies in the first iteration. Each galaxy was fitted twice, using as first guesses for the second iteration the output parameters of the first iteration. Roughly $16 \%$ of the detected galaxies could not be fitted satisfactorily with a single component, but required simultaneous fits with very close companions or multiplecomponent fits. These can be divided in two categories. $9 \%$ of the total are interacting galaxies or very close pairs, where the overlap of the isophotes from different objects required a simultaneous fit. A further $7 \%$ of the total are galaxies for which a single-component Sérsic profile was not sufficient to fit the light profile, leaving significant residuals in the subtraction. Half of these two-component galaxies were re-fit using a disk component and an elliptical bulge, while the other half were re-fit by adding a central point source to the Sérsic component.

As we have shown by the detailed simulations in Paper I, the morphological parameters of the galaxies detected at the depths of our images can be derived with low uncertainties up to $K_{\mathrm{s}} \sim 20.5$, while for fainter objects the uncertainties grow as a function of the magnitude. In addition, we recall that it is possible to set a threshold of $n=2$ on the Sérsic index that can discriminate between late-type galaxies $(n<2)$ and earlytype galaxies $(n>2)$. The results of our simulation are confirmed e.g. by Ravindranath et al. (2004), who used GALFIT to fit single Sérsic profiles to a sample of nearby galaxies of known morphology from the Frei et al. (1996) sample, after artificially redshifting them to $z=0.5$ and $z=1.0$. They found that $n=2$ is the appropriate threshold to separate disk-dominated galaxies from bulge-dominated ones, even in the presence of morphological complexities such as dust, star-forming regions, etc. (Ravindranath et al. 2004).

Of the 383 galaxies detected to $K_{\mathrm{s}} \sim 23.5$ (see Sect. 7 for a discussion of the 112 stars), 214 were classified as late-type and 169 as early-type. The sources fitted with multiple components are classified according to the Sérsic component providing the higher flux contribution. The galaxies are divided in these two subclasses for the following analysis, with an average contamination between the two subclasses of less than $10 \%$ up to $K_{\mathrm{s}}=21$ (Paper I). In order to quantify the morphological fit quality, we used the $\chi_{v}^{2}$ calculated by GALFIT. We classified as well-fit the 315 galaxies with $\chi_{v}^{2} \leq 2$ (167 latetype and 148 early-type), while the other fits were considered less reliable and are not considered when computing the sizemagnitude relation of the galaxies in the SWAN fields (although they are included in the number counts). As an additional check of our late/early-type separation, we show in Fig. 2 the distribution of the axis ratios $b / a$ of the galaxies with $\chi_{v}^{2} \leq 2$ as a function of Sérsic index $n$. As expected, while the late-type galaxies are observed at random inclinations with respect to the plane of the sky, and therefore at every $b / a$, early-type galaxies are not observed with $b / a \lesssim 0.4$ (e.g. Lambas et al. 1992). This confirms that our morphological classification of early and 


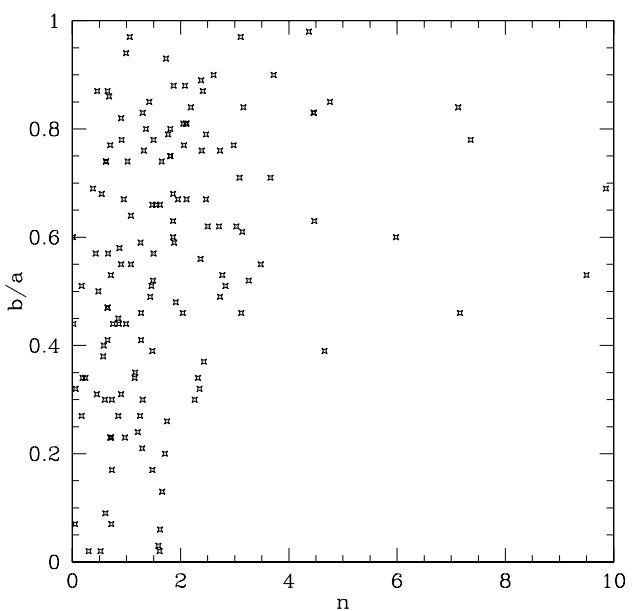

Fig. 2. The distribution of the axis ratios $b / a$ of the SWAN galaxies fitted by GALFIT with $\chi_{v}^{2} \leq 2$ as a function of their Sérsic index $n$. As expected, while late-type galaxies $(n<2)$ are observed at random inclinations with respect to the plane of the sky, and therefore at every $b / a$, early-type galaxies $(n>2)$ are not observed with $b / a \lesssim 0.4$.

late type galaxies based on the Sérsic index $n$ produces reliable results.

While the redshifts of these objects are presently unknown, the magnitude-redshift relation of Cowie et al. (1996) and the K20 survey (Cimatti et al. 2002) indicate that at $K=20$ the median redshift is $z \sim 0.8-1$. At this redshift, our spatial resolution of $0.1^{\prime \prime}$, which also corresponds to the smallest effective radius bin, is equivalent to only $500 \mathrm{pc}$ for typical cosmologies, hinting at the exciting potential of this work.

\section{Star-galaxy separation}

The separation of Galactic foreground stars from the field galaxies is a critical step for avoiding star contamination in our galaxy catalogue. We classified as stars all 58 sources detected in the NACO images with SExtractor stellarity index SSI $\geq 0.9$. The SExtractor classification should be treated with caution since it assumes a constant PSF across each field, and elongated sources are more likely classified as galaxies. However, all the objects classified as stars by SExtractor lie on an upper envelope in a Strehl versus radial distance plot, i.e., they have the highest Strehl ratio among the sources at the same distance from the guide star, supporting their classification as point sources. It remains possible that some stars are not classified as point sources by SExtractor, due to the limited isoplanatic AO patch and their resulting elongated shape. We therefore also classified as stars all the very compact sources fitted by GALFIT with $R_{\mathrm{e}}<0.01^{\prime \prime}$. This is supported by simulations in which we fitted true fiducial point sources in the SWAN fields, rescaled to several magnitudes, with GALFIT Sérsic profiles and obtained very compact effective radii $R_{\mathrm{e}}<0.01^{\prime \prime}$ and high Sérsic indexes. For very bright and elongated PSFs, the fitted $R_{\mathrm{e}}$ can still be as large as $\sim 0.2^{\prime \prime}$, due to the higher signal in the halos of the PSF that may not be perfectly reproduced by the PSF model. We therefore include in the star catalogue all the sources with $K_{\mathrm{S}} \leq 18.5$ (in order to have sufficiently high $\mathrm{S} / \mathrm{N}$ ) that are classified as stars by SExtractor in our SOFI seeing limited images of the SWAN fields. All the objects classified as stars in the seeinglimited images proved to be compact in the AO-corrected ones as well, even if elongated, with all having $R_{\mathrm{e}}<0.16^{\prime \prime}$ as fitted by GALFIT using the appropriate local PSFs for convolution.

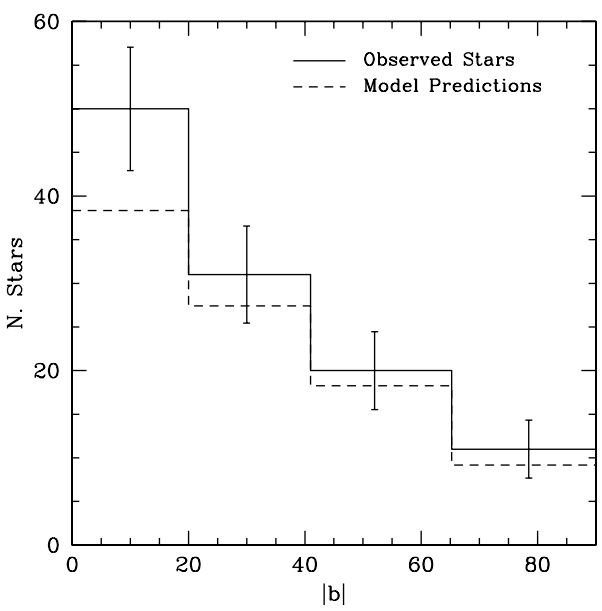

Fig. 3. Number of stars in the SWAN fields as a function of Galactic latitude $b$ up to $K_{\mathrm{s}}=22$, compared with the predictions of the Galaxy model of Bahcall et al. (1980). The observed and predicted stars number counts are in very good agreement for all latitudes except the lowest latitude bin $\left(|b|<20^{\circ}\right)$, where the Galactic model is less accurate due to the high variability between adjacent lines of sight.

We have a total of 112 stars in the 21 SWAN fields analyzed. To assess the robustness of the star/galaxy classification, the star counts were compared with the predictions of the Bahcall et al. (1980) galaxy model, which provides the star counts as a function of the field's Galactic longitude and latitude. As the model provides the number of stars brighter than a certain limiting magnitude in the $V$ band, we convert the $V$ magnitude into a $K_{\mathrm{s}}$ magnitude using an average color derived from the $K$-band counts at the Galactic pole provided by Hutchings et al. (2002). In Fig. 3 we show the number of stars in the SWAN fields as a function of Galactic latitude $b$ up to $K_{\mathrm{s}}=22$, which corresponds to the limit where we are $100 \%$ complete for point sources (see Fig. 5). It can be seen that the observed and predicted stellar number counts are in very good agreement for all latitudes except the lowest latitude bin $\left(|b|<20^{\circ}\right)$, where the Galactic model is less accurate due to the high variability between adjacent lines of sight. However, the total excess of selected stars with respect to the model predictions is only 18 sources, i.e., small compared to the total catalogue of 383 galaxies. Therefore, even if some compact galaxies in these fields were erroneously classified as stars, they represent less than $5 \%$ of the sample.

\section{Completeness correction and number counts}

The probability of detecting a source in one of our images depends on five different parameters:

1. The total integrated magnitude

2. The Sérsic index $n$, as for a given magnitude more concentrated objects (i.e., early-like galaxies with $n>2$ ) are more easily detected than exponential-like galaxies $(n<2)$ with lower concentration.

3. The effective radius $R_{\mathrm{e}}$. As before, more compact sources are more easily detected.

4. The SWAN field in which the source was observed. The integration time and therefore the signal/noise ratio is different in different fields. In addition the overall AO correction is different in each observation, as is indicated by the different on-axis Strehl ratios (see Table 1). 

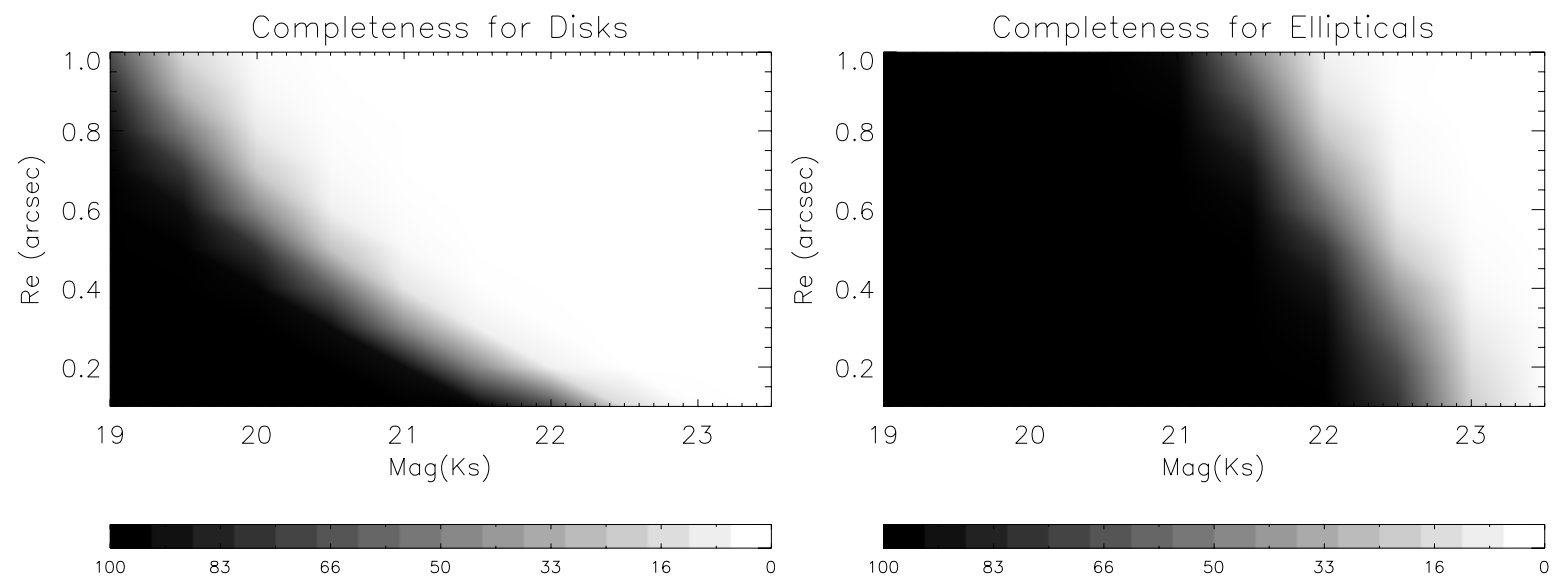

Fig. 4. The left panel shows the variation of the detection probability for a late-type galaxy in SWAN as a function of the magnitude and the effective radius $R_{\mathrm{e}}$. The probability is the average for all 21 observed fields, and assumes a distance from the guide star $1<\theta / \theta_{0} \leq 2$. The right panel shows the same for early-type galaxies.

5. The distance from the guide star $\theta / \theta_{0}$, as the degree of correction of the AO system depends strongly on this parameter (see, e.g., Paper I).

The last two parameters are due to the distinctive attributes of our survey, which makes use of several different fields (4) and of $\mathrm{AO}(4,5)$.

In order to derive the detection probability for each combination of these five parameters, we ran several simulations, adding a total of 65000 simulated galaxy profiles with known parameters - matched to the ones of the observed galaxies - to the original SWAN fields at random locations and tried to recover them running SExtractor again. We used extended sources to evaluate the completeness correction, as this produces results that are quite different from those inferred using point sources alone, especially at this resolution. SExtractor was used with the same parameters used in the science source detection. We used the Sérsic index $n=1$ for late-type galaxies and $n=4$ for early-type galaxies. For both types the effective radius $R_{\mathrm{e}}$ ranged from $0.1^{\prime \prime}$ to $1.0^{\prime \prime}$. The galaxy profiles were convolved with real NACO PSFs extracted from point sources in our data lying at different distances from the guide star, in order to simulate the effect of the AO correction. The simulated galaxies have magnitudes ranging between $K_{\mathrm{s}}=19$, where we are $100 \%$ complete for every combination of the other four parameters, to $K_{\mathrm{s}}=23.5$. We consider three different regimes for the detection probability as a function of the distance from the guide star: $\theta / \theta_{0} \leq 1$, $1<\theta / \theta_{0} \leq 2$ and $\theta / \theta_{0}>2$. We used point sources at $\theta / \theta_{0}=0.9$, 1.5 , and 2.8 respectively for the three regimes as references for the PSF in the simulated galaxies.

Using this approach, we can derive the detection probability for a galaxy of known magnitude, $R_{\mathrm{e}}$, Sérsic index, and distance from the guide star in a particular field. By way of example, the histograms of the detection probability averaged over all 21 fields as a function of magnitude and of effective radius $R_{\mathrm{e}}$ for late and early-type galaxies with $1<\theta / \theta_{0} \leq 2$ are shown in Fig. 4. It is clear from comparing the panels how much more sensitive high resolution images are to more core-concentrated sources like the elliptical galaxies.

The detection probability can be used to correct the number counts using the observed galaxy population as a starting point. From our simulations we derived the detection probability $P_{\text {detect }}$ for each detected galaxy in the survey, using the measured $R_{\mathrm{e}}$, magnitude, and Sérsic index from GALFIT fitting (see Sect. 6),

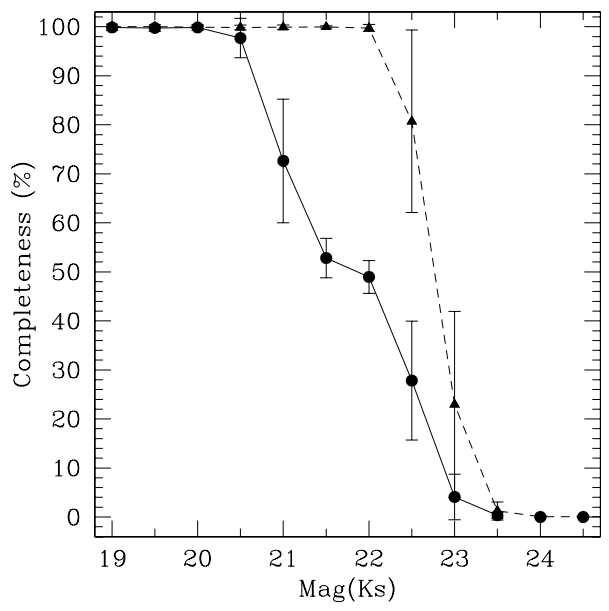

Fig. 5. Comparison between the completeness for point sources and for extended sources for the SWAN fields. The completeness for point sources (triangles, dashed line) was evaluated adding 100 true NACO point sources $\left(\theta / \theta_{0}=1.5\right)$ for each magnitude to each field and then averaging over all fields. The completeness for extended sources (circles, solid line) is the average over all the fields for both late and early-type with $R_{\mathrm{e}}=0.3^{\prime \prime}$, i.e., the mean for all the detected sources in SWAN, at the same distance from the guide star used for the point sources. The errorbars show the variance for the 21 different fields.

along with the position of the galaxy in the field. Each galaxy is then regarded in the completeness-corrected number counts as $1 / P_{\text {detect }}$ sources at its magnitude, so that, for example, a galaxy with $P_{\text {detect }}=0.80$ counts as 1.25 galaxies once the correction is applied.

In Fig. 5 we show the average completeness over all the fields for both point sources and extended objects as a function of magnitude. The completeness for point sources was derived by adding (100times) to each field a true NACO point source with rescaled flux. The point source was at a distance $\theta / \theta_{0}=1.5$ from the guide star. The completeness for extended objects is the average between that of late-type and of early-type galaxies over all the fields, using the same NACO point source PSF to convolve the simulated galaxy profiles. The effective radius was fixed at the average for the detected SWAN sources $\left(R_{\mathrm{e}}=0.3^{\prime \prime}\right)$. Obviously the correction derived using only point sources is much smaller than the one derived as described above, with the number of sources in the range $20.5 \leq K_{\mathrm{s}} \leq 22$ (where no 
Table 2. Differential number counts in the SWAN fields. The raw number of detected galaxies is reported in (2). In (3) the corrected number counts $\left(\mathrm{N} \mathrm{mag}^{-1} \mathrm{deg}^{-2}\right)$ for the whole sample are shown, while (4) and (5) separate late-type and early-type counts, respectively.

\begin{tabular}{ccccc}
\hline \hline $\begin{array}{c}\operatorname{Mag}\left(K_{\mathrm{s}}\right) \\
(1)\end{array}$ & $\begin{array}{c}n_{\text {raw }} \\
(2)\end{array}$ & $\begin{array}{c}N_{\text {tot }} \\
(3)\end{array}$ & $\begin{array}{c}\text { Late-type } \\
(4)\end{array}$ & $\begin{array}{c}\text { Early-type } \\
(5)\end{array}$ \\
\hline 14 & 3 & $7 \mathrm{e}+02$ & $2 \mathrm{e}+02$ & $5 \mathrm{e}+02$ \\
15 & 8 & $1.9 \mathrm{e}+03$ & $5 \mathrm{e}+02$ & $1.4 \mathrm{e}+03$ \\
16 & 8 & $1.8 \mathrm{e}+03$ & $1.4 \mathrm{e}+03$ & $4 \mathrm{e}+02$ \\
17 & 19 & $4 \mathrm{e}+03$ & $1.4 \mathrm{e}+03$ & $3.0 \mathrm{e}+03$ \\
18 & 62 & $1.5 \mathrm{e}+04$ & $4.9 \mathrm{e}+03$ & $9 \mathrm{e}+03$ \\
19 & 69 & $1.6 \mathrm{e}+04$ & $9 \mathrm{e}+03$ & $7.8 \mathrm{e}+03$ \\
20 & 96 & $2.8 \mathrm{e}+04$ & $2.0 \mathrm{e}+04$ & $8.7 \mathrm{e}+03$ \\
21 & 67 & $4.8 \mathrm{e}+04$ & $4.1 \mathrm{e}+04$ & $6.8 \mathrm{e}+03$ \\
22 & 35 & $9.8+04$ & $9.3 \mathrm{e}+04$ & $4.4 \mathrm{e}+04$ \\
\hline
\end{tabular}

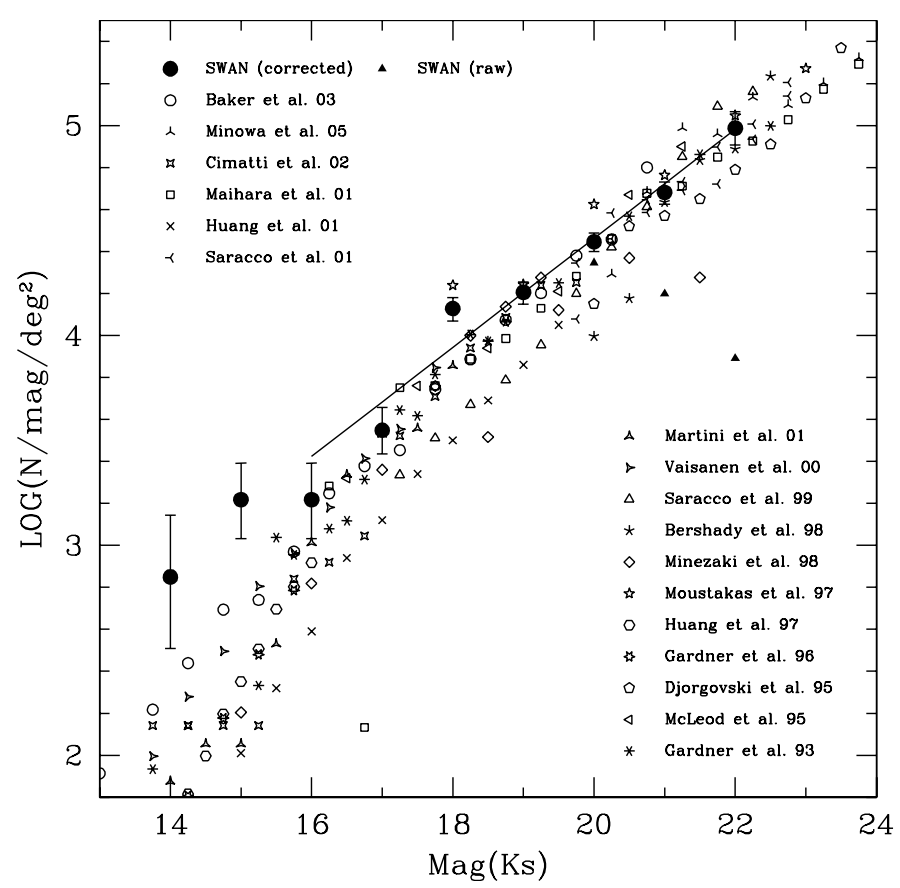

Fig. 6. $K_{\mathrm{s}}$ corrected (solid circles) and uncorrected (solid triangles) number counts from SWAN, compared with other $K$-band surveys. The points at $K_{\mathrm{s}} \geq 23$ are not reliable as the completeness correction is adding more than $90 \%$ of the sources. The excess at bright magnitudes is due to a selection bias, as some of the fields were selected to contain more bright galaxies (Baker et al. 2003). The solid line is the best fitting power-law in the range $16 \leq K_{\mathrm{s}} \leq 22$, with a slope $\mathrm{d} \log (N) / \mathrm{d} M=0.26$. The error bars show the Poissonian errors.

correction would be applied in the point-source case) being particularly underestimated.

The corrected number counts, obtained using the detection probabilities of the observed SWAN sources as weights, are shown in Fig. 6. At brighter magnitudes $\left(K_{\mathrm{s}} \leq 16\right)$, an excess is present in our number counts with respect to others in the literature. This is due to a selection bias, as some of the fields were chosen to have an excess of bright galaxies (see Baker et al. 2003). At fainter magnitudes, the points at $K_{\mathrm{s}} \geq 23$ are no longer reliable, as the completeness correction adds more than $90 \%$ of the sources. We derive a slope $\mathrm{d} \log (N) / \mathrm{d} M=0.26 \pm 0.01$ in the range $16 \leq K_{\mathrm{s}} \leq 22$. Our corrected number counts are in good agreement with those found by other authors in the literature (see Fig. 6). This result, together with what was found in
Paper I, shows once more that we are able to account for the biases introduced by the variable AO PSF and that the data can therefore be reliably used for further analysis.

\section{Comparison with galaxy evolution models}

Most theoretical models of galaxy formation and evolution can be roughly divided into two broad categories: the so-called "backwards" approach and the "ab initio" approach. In the former approach (Tinsley 1980; Yoshii \& Takahara 1988; Fukugita et al. 1990; Rocca-Volmerange \& Guiderdoni 1990; Yoshii \& Peterson 1995; Pozzetti et al. 1996, 1998; Jimenez \& Kashlinsky 1999; Totani \& Yoshii 2000; Totani et al. 2001), the evolution is probed backwards into the past to predict observables such as galaxy counts and redshift distributions. The local properties of galaxies, like multi-band colors and chemical properties, are used to construct a reasonable model of the star formation history and luminosity evolution of galaxies based on the stellar population synthesis method. The formation epoch and merging history of galaxies, however, cannot be predicted in this framework, as they are introduced as phenomenological parameters that can be inferred from comparison with observational data.

In the latter approach (Kauffmann et al. 1993; Cole et al. 1994, 2000; Somerville \& Primack 1999; Nagashima et al. 2005), on the other hand, the formation epoch and merging history of galaxies are predicted by the standard theory of structure formation in the CDM universe. In these models the local and high redshift properties of the galaxies such as the luminosity function, mass and size distribution, are outputs of the model that can be compared with observations. However, although the formation and evolution of dark matter halos are considered to be rather well understood, our knowledge about baryonic processes such as star formation, supernova feedback, and galaxy merging is still very poor, and a number of phenomenological parameters must be taken into account, making the comparison of the modeled and observed data more complex. Here we compare the galaxy counts in SWAN and the size-magnitude relation of the detected galaxies with representative results of these two radically different approaches.

\subsection{The galaxy evolution models}

The first model used is a "backwards" pure luminosity evolution (PLE) model developed by Totani \& Yoshii (2000) and Totani et al. (2001), based on the present-day properties of galaxies and their observed luminosity function. It evolves a system's luminosity and spectral energy distribution evolution backward in time using a standard galaxy evolution model in which star formation is tuned to reproduce galaxies' present-day colors and chemical properties (Arimoto \& Yoshii 1987; Arimoto et al. 1992). The model includes the effects of both internal dust obscuration and intergalactic $\mathrm{H} \mathrm{I}$ absorption, and it does not incorporate galaxy mergers; therefore the galaxy sizes and comoving number density do not evolve. The number density of galaxies is normalized at $z=0$ using the local $B$-band luminosity function of galaxies, while the relation of the present $B$ luminosity and effective radius $R_{\mathrm{e}}$ is determined from power-law fits to the empirical relation observed for local galaxies of different types (Bender et al. 1992; Impey et al. 1996). Galaxies are in fact classified into six morphological types: three of them (Sab, Sbc, and Scd) are assigned to spiral galaxies, while an Sdm model is used for irregular galaxies. Following Totani et al. (2001), we divided the E/S0 galaxies into distinct population of giant ellipticals (gE, $\left.M_{B} \lesssim-17\right)$ and dwarf ellipticals (dE, $\left.M_{B} \gtrsim-17\right)$. It is 
known that these are two distinct populations, showing different luminosity profiles (the $r^{1 / 4}$ law for giants and exponential for dwarfs; see Barazza et al. 2005), different luminosity-size relations, luminosity functions and different physical processes that govern the evolution of each type (see, e.g., Ferguson \& Binggeli 1994 and references therein; Ilbert et al. 2006 for evidence of two different populations up to $z \sim 1.2$ ).

In the $K_{\mathrm{s}}$ band, the critical separation magnitude $\left(M_{B}=-17\right)$ corresponds to $M_{K_{\mathrm{s}}} \sim-21$ for the typical color of elliptical galaxies. Since the contribution of early-type galaxies is more significant in the near-infrared than in the optical, it is important to take into account such distinct populations of elliptical galaxies in predicting the $K_{\mathrm{s}}$ counts.

In addition, we compare the derived properties of the SWAN galaxies with the predictions of the "ab initio" Numerical Galaxy Catalog (NuGC) of Nagashima et al. (2005), which is based on a semi-analytic (SA) model of galaxy formation combined with high-resolution $N$-body simulations in a $\Lambda \mathrm{CDM}$ cosmological framework. The model includes several essential ingredients for galaxy formation, such as the merging histories of dark halos directly derived from $N$-body simulations, radiative gas cooling, star formation, supernova feedback, mergers of galaxies, population synthesis, and extinction by internal dust and intervening H I clouds. The high resolution used for the simulations, with a minimum mass for dark halos of $3 \times 10^{9} M_{\odot}$, is sufficient to resolve their effective Jeans mass.

It was shown by Nagashima et al. (2005) that this model is in reasonable agreement with several observational results, like the luminosity functions in $B$ and $K$ bands, the H I mass function, the size-magnitude relations for local spirals and elliptical galaxies, the Tully-Fisher and Faber-Jackson relations at $z=0$, faint galaxy number counts in $B V R i^{\prime} z^{\prime}$ bands, and the cosmic star formation history at high redshift. In addition, the model is able to reproduce the distribution of $(R-K)_{A B}$ colors with redshift observed in GOODS (Somerville et al. 2004), including extremely red $\left(\left(R-K_{\mathrm{s}}\right)_{A B} \sim 5\right)$ galaxies that other semi-analytic treatments have trouble accounting for. In summary, the model is able to reproduce several observational results for local and high-redshift galaxies, not just those that were used to tune the model parameters.

\subsection{Addressing cosmic variance}

The uncertainties in galaxy number counts include contributions from Poisson errors and from the so-called "cosmic variance", due to the fact that galaxies are strongly clustered and thus distributed in overdensities and large voids on the sky. Therefore, we have to take into account the corresponding effects on the relative normalizations of the predicted and observed counts in order to make a fair comparison. According to Daddi et al. (2000), the RMS fluctuations $\sigma_{\text {tot }}$ of numbers counts around their mean mean value, taking into account both the Poisson error and the cosmic variance, are given by

$\sigma_{\text {tot }}^{2}=X \cdot(1+X A C)$

where $X$ are the total number counts, $A$ is the clustering amplitude $\left(A \sim 1.6 \times 10^{-3}\right.$ for the Daddi et al. (2000) $K$-selected sample), and $C$ is a factor that depends on the field area and can be approximated by $C=58 \mathrm{area}^{-0.4}$ for an area expressed in $\operatorname{arcmin}^{2}$. In our case, as we observe galaxies in $N=21$ different fields of the same area, thus reducing the clustering uncertainty, Eq. (2) can be written as

$\sigma_{\text {tot }}^{2} \simeq X_{\text {tot }} \cdot\left(1+\frac{X_{\text {tot }}}{N} A C\right)$ where $N$ is the number of fields, $X_{\text {tot }}$ is the total number of sources detected in all the fields, and $C$ corresponds to the area of a single field. Therefore we derive that the relative uncertainty on the number counts is given by

$\frac{\sigma_{\mathrm{tot}}}{X_{\mathrm{tot}}}=\sqrt{\frac{1}{X_{\mathrm{tot}}}+\frac{A C}{N}}=0.08$

for the SWAN fields, while the contribution from Poisson statistics would only be of the order of $5 \%$.

We expect that the predictions of any successful model should fit the observed SWAN counts within a discrepancy of this order of magnitude. In good agreement with these expectations, we find that a correction of $4 \%$ produces the best match with the total number counts in the PLE model, while a correction of $1 \%$ provides the best match to the hierarchical model. The model predictions discussed in the following sections have been renormalized accordingly, i.e., PLE and hierarchical model normalizations are multiplied by 1.04 and 1.01 respectively.

\subsection{Comparison of the SWAN data with the model predictions}

The predictions of the PLE model for the galaxy counts and galaxy size as a function of the $K_{\mathrm{s}}$ magnitude were compared with the number counts and effective radii $R_{\mathrm{e}}$ observed in the SWAN images. We are assuming for the PLE model $H_{0}=$ $70 \mathrm{~km} \mathrm{~s}^{-1} \mathrm{Mpc}^{-1}, \Omega_{\mathrm{m}}=0.27, \Omega_{\Lambda}=73$, and a formation redshift $z_{F}=5$ for all galaxy types (Totani et al. 2001). The comparison between the PLE model and the total completeness corrected number counts is shown in Fig. 7a. As explained in Sect. 8, the points at $K_{\mathrm{s}} \leq 15$ show an excess due to a selection bias, as some fields were selected to contain more bright galaxies. Counts from other surveys in the literature for $K_{\mathrm{s}} \leq 16$ were therefore plotted for comparison to the model in this range, and good agreement is found between the SWAN galaxy counts and the PLE predictions.

Figure $7 \mathrm{~b}$ shows the average effective radius $\left\langle\log \left(R_{\mathrm{e}}\right)\right\rangle$ of the galaxies with the most reliable morphological fitting, i.e., those fit by GALFIT with $\chi_{v}^{2} \leq 2$. The average must take into account the selection effects due to the different detection probabilities of the galaxies. We therefore weighted each galaxy using its detection probability as derived in Sect. 8. The error bars show the standard error on the mean. The observed data points are compared with the predictions of the PLE model, which manifests a slight overprediction of the galaxies' $R_{\mathrm{e}}$. This effect is mainly due to the late-type galaxy population (see Fig. 10), for which there might be hint of an increase in size, and will be discussed in Sect. 9.4. Our results confirm the finding of Totani et al. (2001) and Minowa et al. (2005), who found in the Subaru Deep Field and Subaru Super Deep Field that a PLE model with no number or size evolution gives the best fit to their $K$-selected sample's number counts and isophotal area distribution.

The NuGC hierarchical model is also compared with the total galaxy counts and $R_{\mathrm{e}}$ distribution for SWAN in Fig. 7. Within the observational uncertainties of the shape of the observed luminosity functions, Nagashima et al. (2005) adopted two different models, characterized by two different supernova feedback regimes- their strong supernova feedback (SSFB) model and weak supernova feedback (WSFB) models. Comparisons with the total faint number counts and isophotal area distribution for $K$-band selected galaxies in the Subaru Deep Field, and with redshift distributions for faint galaxies, showed that the SSFB model is in better agreement with the properties of the $K$-selected sample (Nagashima et al. 2002; Nagashima et al. 2005), although the 

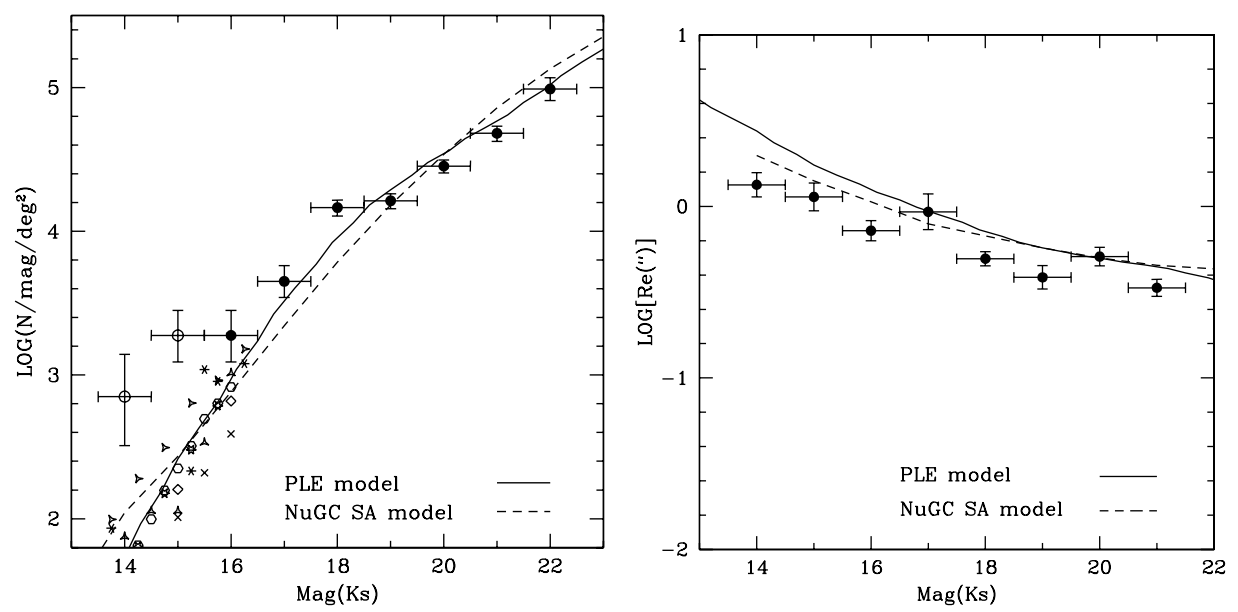

Fig. 7. Comparison of total number counts for all galaxies (left panel), and of the mean $R_{\mathrm{e}}$ as a function of magnitude for those sources with $\chi_{v}^{2} \leq 2$ in the GALFIT fit (right panel), with the PLE model (solid line) and the hierarchical model (dashed line) predictions. In the number counts, the points at $K_{\mathrm{s}} \leq 15$ shows an excess due to a selection bias, as some fields were selected to contain more bright galaxies. Counts from other surveys in the literature for $K_{\mathrm{s}} \leq 16$ were therefore plotted for comparison to the model in this range (see Fig. 6 for the references of the data points). The error bars on the number counts are the Poisson error, while in the size-magnitude relation they represent the standard error on the mean.
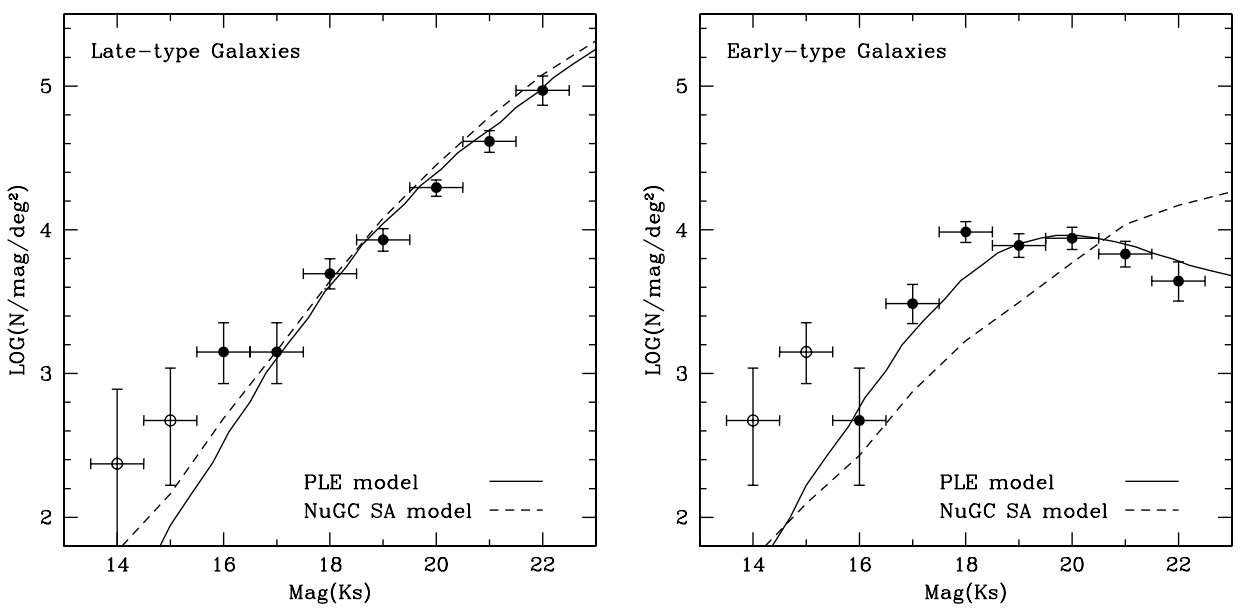

Fig. 8. Comparison of the SWAN counts for exponential profile galaxies $(n<2$, left panel $)$ and early-type galaxies $(n>2$, right panel) with the PLE model (solid line) and hierarchical model (dashed line) predictions. The open circles are not reliable points, as explained in the legend of Fig. 7.

predicted counts were overestimated for $K \gtrsim 23$. We therefore compare the properties of the SWAN galaxies with the SSFB model predictions only in the following. A $\Lambda$ CDM cosmology is used for the hierarchical model, with $\Omega_{\mathrm{m}}=0.3, \Omega_{\Lambda}=0.7$ and $H_{0}=70 \mathrm{~km} \mathrm{~s}^{-1} \mathrm{Mpc}^{-1}$. As found by Nagashima et al. (2005), the model is in good agreement with the total number counts data, and matches the $R_{\mathrm{e}}$ distribution in SWAN as well as the PLE model.

\subsection{Morphological type dependent comparison}

In addition to the total number counts and size-magnitude relation, our high-resolution $\mathrm{AO}$ morphological classification of the SWAN galaxies allows us to assess the predictions of the models for the counts and sizes of the late type and early type galaxies separately. This is one of the first times such a comparison has been done in the near-IR, as both AO observations and accurate PSF modeling are needed to obtain reliable morphological classification of faint field galaxies at these wavelengths. We are therefore able to compare our observational data with untested predictions of the two models.
In Fig. 8a, the PLE model predictions for the number counts of all galaxies with exponential profiles (spirals, irregulars and dwarf ellipticals) are compared with the observations for galaxies with Sérsic index $n<2$ according to GALFIT. We find good agreement between model and data for the number counts, as before. A very similar result is found using the hierarchical model prediction for late-type galaxies.

Fig. $8 \mathrm{~b}$ shows the predictions of both models for early-type galaxies, compared with the observations for $n>2$ galaxies in SWAN. In the SWAN data, the elliptical counts are much flatter than the late-type counts, showing a plateau for $K_{\mathrm{s}}>20$. A much flatter slope in the early-type galaxies number counts with respect to the late-types was found also by Teplitz et al. (1998), using HST NICMOS observations in the $H$ band, and in deep optical observations (e.g. Abraham et al. 1996). The adopted PLE model is able to convincingly reproduce the plateau observed in the counts for $K_{\mathrm{s}}>20$; a similar behavior is predicted by other PLE models (see, e.g., Pozzetti et al. 1996). This plateau is produced in the model by a combination of two effects. First, the luminosity function of the $\mathrm{gE}$ population is bell-like (see, e.g., Totani et al. 2001), and the number of faint gEs decreases rapidly 
with decreasing luminosity. Second, in the PLE model beyond $z>1.5$ giant ellipticals are very faint, due to heavy extinction in the model ( $\tau \gtrsim 10)$, as described in Totani \& Yoshii (2000). As a result, for $K_{\mathrm{s}} \geq 20$ (corresponding to an $L^{*}$ galaxy at $z=1.5$ ), going to fainter magnitudes does not correspond to an increase of the sampled volume. Instead, beyond $K_{\mathrm{s}}=20$, the model predicts we should begin to miss the dusty high-redshift progenitors of today's ellipticals, consistent with the plateau observed in the SWAN data. A scenario in which massive $z \gtrsim 1.5$ ellipticals are highly obscured by dust during their starburst phase, and therefore produce the plateau observed in our $K$-band number counts, is consistent with the detection of very luminous, highly obscured submillimeter galaxies at high redshift (Blain et al. 2002, and references therein). In addition, galaxies with unusually red IR colors that have been measured in deep in nearIR observations can be explained as primordial elliptical galaxies that are reddened by dust and still in the starburst phase of their formation at $z \gtrsim 3$ (e.g., Totani et al. 2001).

In contrast to the success of the PLE model, the hierarchical one considerably underpredicts at bright magnitudes $\left(K_{\mathrm{s}} \lesssim 20\right)$ and overpredicts at fainter magnitudes the observed number counts. In particular, the observed plateau in the early-type counts, which was very well reproduced in the PLE model, is not expected at all in the hierarchical model predictions. This disagreement implies that the processes that produce an elliptical galaxy, in at least this particular hierarchical model, are not adequate to describe reality. In the model, an elliptical galaxy is formed through a major merger, i.e., a merger with mass ratio $f=m_{1} / m_{2} \geq 0.3$, in which it is assumed that all the cold gas turns into stars and hot gas, and all the stars populate the bulge of a new galaxy. Although it may be possible to increase the number of bright ellipticals by changing the model parameter that regulates the mass ratio distinguishing major from minor mergers, it seems harder to decrease the model's number of $K_{\mathrm{s}}>20$ ellipticals to the level observed in SWAN (Nagashima private communication). In this case no separation is possible between the $\mathrm{gE}$ and $\mathrm{dE}$ populations, as all the major mergers produce galaxies with a de Vaucouleurs profile.

We note that misclassification of galaxies between the two categories of early and late-type is not expected to strongly affect these conclusions. We expect only $\sim 10 \%$ of late-type to be misclassified as early-type and $\sim 10 \%$ of early-type to be misclassified as late-type at $K_{\mathrm{s}}=21$ (see Paper I). Since at faint magnitudes the number counts of spirals are $\sim 10$ times higher than those of ellipticals, it is more likely that we are overestimating the number of faint ellipticals in our sample. Correcting for this bias would make the discrepancy with the hierarchical model even larger.

We conclude that the PLE model better reproduces the observed number counts of the SWAN galaxies. In Fig. 9 the contributions of the different galaxy types to the total observed number counts according to the PLE model are shown. The separation between the two populations of $\mathrm{dE}$ and $\mathrm{gE}$ galaxies proves to be a key element in reproducing the number counts separated by morphological type on the basis of their light profiles. In fact, the number counts for exponential-like profiles would have been underestimated for $K_{\mathrm{s}} \gtrsim 20$, and the $r^{1 / 4}$ profiles number counts overestimated, had the $\mathrm{dE}$ galaxies been included in the early-type morphological bin along with the gE galaxies. This result confirms the suggestions of Totani et al. (2001) that such a separation better reproduced the observed number counts in the Subaru Deep Field for $K \gtrsim 20$ than a model with a single elliptical population.

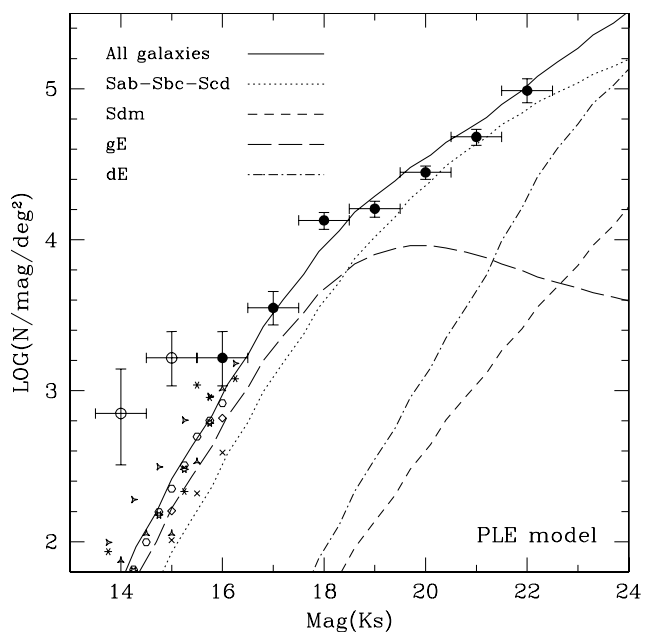

Fig. 9. Comparison of the total SWAN number counts with the PLE model predictions. The contributions of different galaxy types are shown as different thin lines, while the prediction for the total population of galaxies is shown as a solid line. The large open circles are not reliable, as explained in the caption of Fig. 7.

In Fig. 10, the observed size-magnitude distribution discriminating between late-type and early-type galaxies is compared with the model predictions. At bright magnitudes, the observed galaxies are smaller than the model predictions for both earlytype and late-type samples. This discrepancy is likely due to an uncorrected systematic effect: large $\left(R_{\mathrm{e}} \gtrsim 1^{\prime \prime}\right)$ bright galaxies can be self-subtracted in the reduction process, as explained in Sect. 4. This phenomenon will reduce the apparent sizes of sources, although their integrated magnitudes (and thus their contributions to the number counts) will be only minimally affected.

At faint magnitudes, the models are able to reproduce the observed distributions for early-type galaxies. However, the PLE model better reproduces the observed distribution at fainter magnitudes, where the hierarchical model predicts more compact objects than are observed. In contrast, an offset may exist between both models and the data for late-type galaxies, which appear $\sim 30 \%$ smaller than predicted at faint magnitudes. This offset is what would be expected for modest growth in the sizes of latetype galaxies. In both of the models considered here, the sizes of disks for a given mass are almost independent of the formation redshift. This property is built into any PLE model, but even the hierarchical model by Nagashima et al. (2005) assumes that there is almost no evolution in the stellar mass-size relation for disk galaxies, as suggested by the observations of Barden et al. (2005) up to $z \sim 1$. If our observed offset is really due to increses in size in the late-type population, the result would be qualitatively consistent e.g. with the predictions of Mo et al. (1998), who estimated that disk galaxies forming at $z=1$ are $50 \%$ smaller than disks forming at $z=0$. However, the inclusion of galaxies with many different redshifts, masses, and $M / L$ ratios in the faint bins of Fig. 10b prevents any robust quantitative conclusion.

Our finding that pure luminosity evolution of galaxies is favored for a $K_{\mathrm{s}}$-selected sample up to $K_{\mathrm{s}} \sim 22$, without evidence of relevant number evolution even when separating between late and early-type galaxies is consistent with other results. For example, Truijllo et al. (2005) used deep near-infrared images from the FIRES survey, combined with GEMS and SDSS data, to confirm that the observed size-magnitude relation evolution out to $z \sim 1.7$ for late-type objects matches very well the expected 

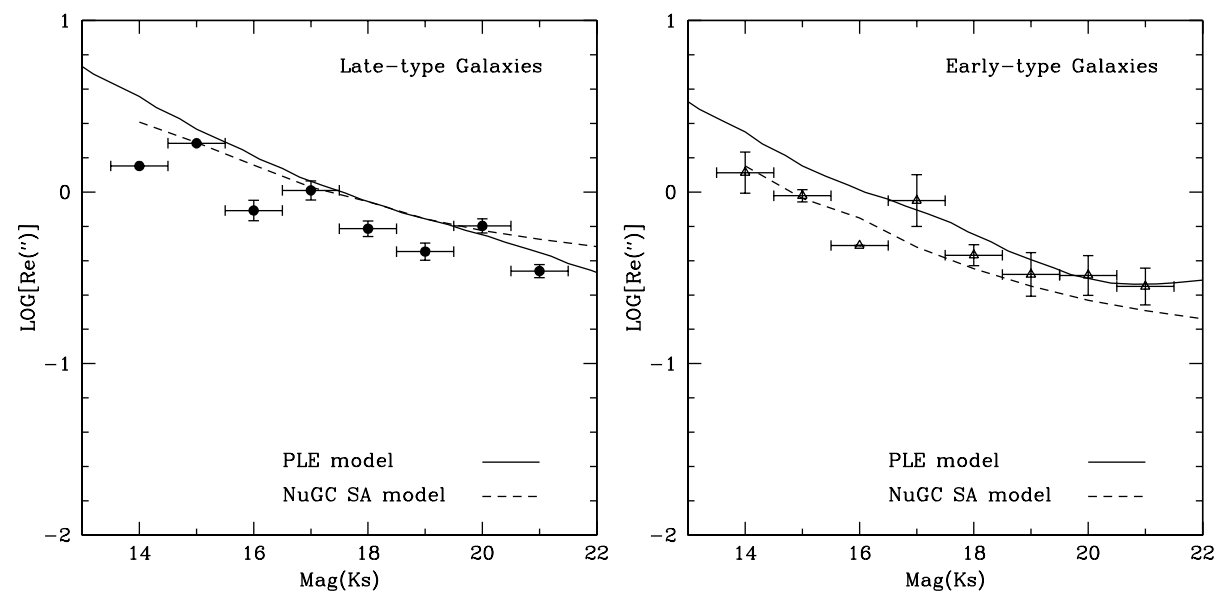

Fig. 10. Comparison of the $R_{\mathrm{e}}$ distribution as a function of magnitude of late-type (left panel) and early-type galaxies (right panel) with $\chi_{v}^{2} \leq 2$ in the GALFIT fit with the PLE model (solid line) and hierarchical model (dashed line) predictions. The error bars are the standard error on the mean; no error bar is drawn for a bin with one galaxy.

evolution for Milky-Way type objects from infall models, while for spheroid-like objects the evolution of the luminosity-size relation was found to be consistent with pure luminosity evolution of a fading galaxy population. McIntosh et al. (2005) studied a large sample of early-type galaxies from the GEMS survey, finding that the luminosity-size and stellar mass-size relations evolve in a manner that is consistent with the passive aging of ancient stellar population. Papovich et al. (2005) suggest that passive evolution can account for the observed luminosity-size relation at $z \sim 1$, with merging becoming important at higher redshifts.

\section{Conclusions}

In this paper we have presented new results from a high resolution adaptive optics assisted morphological study of 21 fields from SWAN, the Survey of a Wide Area with NACO. The PSF model derived in Paper I was used in combination with GALFIT to classify the SWAN galaxies into the two classes of early and late type, and to derive effective radii $R_{\mathrm{e}}$ of 383 galaxies. A detailed study of the detection probability as a function of the magnitude, Sérsic index, effective radius, field and distance from the guide star was performed in order to take careful account of the selection effects affecting our sample. The results were used to compute the completeness-corrected number counts and to derive the average $R_{\mathrm{e}}$ as a function of magnitude.

The number counts and size-magnitude relation for the total galaxy population, and for early and late-type separately, were compared with both a modified version of the pure luminosity evolution model of Totani \& Yoshii (2000) and with the a priori hierarchical model developed by Nagashima et al. (2005). We have shown in Sect. 9 that while the hierarchical model can convincingly reproduce the counts of late-type galaxies, it is not consistent with the observed number counts of elliptical galaxies selected in the $K_{\mathrm{s}}$ band. On the other hand, the PLE model can reasonably reproduce both the late and early type count distributions for the SWAN galaxies. We have compared the sizemagnitude distribution of the galaxies with the predictions of the models, finding that there might be some hint of increased size for the late-type galaxy population. Both models are consistent with the observed distribution for early-type galaxies, although the PLE model seems to better reproduce the observed distribution at fainter magnitudes. Our work therefore favors pure luminosity evolution of early-type galaxies for a $K_{\mathrm{s}}$-selected sample up to $K_{\mathrm{s}} \sim 22$. In contrast, our results show that a representative example of currently available models based on the hierarchical galaxy formation theory is not able to reproduce the observed properties of faint $K_{\mathrm{s}}$-selected early-type galaxies in the near-IR.

These results illustrate the importance of obtaining reliable morphological classifications for better constraining the details of galaxy formation and evolution models, and demonstrate the unique power of $\mathrm{AO}$ observations to extend such work to faint galaxies in the near-IR.

Acknowledgements. We thank the anonymous referee for useful comments and suggestions. The authors are grateful to the staff at Paranal Observatory for their hospitality and support during the observations. We thank Masahiro Nagashima for providing the $K$-band simulated data from the Numerical Galaxy Catalog and useful discussion of our results; Reinhard Genzel, Reiner Hofmann, Sebastian Rabien, Niranjan Thatte, and W. Jimmy Viehhauser, for their help and discussion of SWAN strategy and results; and Rainer Schödel for the observations of SBSF 41. Some of the data included in this paper were obtained as part of the MPE guaranteed time programme. G.C. and A.J.B. acknowledge MPE for support; A.J.B. acknowledges support from the National Radio Astronomy Observatory, which is operated by Associated Universities, Inc., under cooperative agreement with the National Science Foundation.

\section{References}

Abraham, R. G., Tanvir, N. R., Santiago, et al. 1996, MNRAS, 279, L47

Arimoto, N., \& Yoshii, Y. 1987, A\&A, 173, 23

Arimoto, N., Yoshii, Y., \& Takahara, F., 1992, A\&A, 253, 21

Bahcall, J. N., \& Soneira, R. M. 1980, ApJS, 44, 73

Baker, A. J., Davies, R. I., Lehnert, M. D., et al. 2003, A\&A, 406, 593

Baker, A. J., Davies, R. I., Lehnert, M. D., et al. 2005, in Science with Adaptive Optics, Proc. ESO workshop, Sept. 2005, ed. M. Kasper, \& W. Brandner, 359

Barazza, F. D., \& Jogee, S. 2005, Near-fields cosmology with dwarf elliptical galaxies, IAU Colloq., 198, 177

Barden, M., Rix, H.-W., Somerville, R. S., et al. 2005, ApJ, 635, 959

Bell, E. F., \& de Jong, R. S. 2001 ApJ, 550, 212

Bender, R., Burstein, D., \& Faber, S. M. 1992, ApJ, 399, 462

Bershady, M. A., Lowenthal, J. D., \& Koo, D. C. 1998, ApJ, 505, 50

Bertin, E., \& Arnouts, S. 1996, A\&AS, 117, 393

Blain, A. W., Smail, I., Ivison, R. J., Kneib, J.-P., \& Frayer, D. T. 2002, Phys. Rep., 369, 111

Bouwens, R. J., Illingworth, G. D., Blakeslee, J. P., Broadhurst, T. J., \& Franx, M. 2004, ApJ, 611, L1

Brinchmann, J., \& Ellis, R. S. 2000, ApJ, 536, L77

Cimatti, A., Pozzetti, L., Mignoli, M., et al. 2002, A\&A, 391, L1

Cole, S., Aragon-Salamanca, A., Frenk, C. S., Navarro, J. F., \& Zepf, S. E. 1994, MNRAS, 271, 781

Cole, S., Lacey, C. G., Baugh, C. M., \& Frenk, C. S. 2000, MNRAS, 319, 168

Connolly, A. J., Szalay, A. S., Dickinson, M., Subbarao, M. U., \& Brunner, R. J. 1997, ApJ, 486, L11

Cowie, L. L., Gardner, J. P., Hu, E. M., et al. 1994, ApJ, 434, 114 
Cowie, L., Songaila, A., Hu, E., \& Cohen, J. G. 1996, AJ, 112, 839

Cresci, G., Davies, R. I., Baker, A. J., \& Lehnert, M. D. 2005, A\&A, 438, 757

Christopher, N. M., \& Smail, I. 2006, MNRAS, 365, 439

Daddi, E., Cimatti, A., Pozzetti, L., et al. 2000, A\&A, 361, 535

Davies, R. I., Lehnert, M., Baker, A. J., et al. 2001, IAU Symp., 205, 455

Davies, R. I., et al. 2006 in preparation

Dickinson, M. 1999, After the Dark Ages: When Galaxies were Young (the Universe at $2<z<5$ ), AIP Conf. Proc., 470, 122

Dickinson, M., Hanley, C., Elston, R., et al. 2000, ApJ, 531, 624

Djorgovski, S., Soifer, B. T., Pahre, M. A., et al. 1995, ApJ, 438, L13

Drory, N., Bender, R., Feulner, G., et al. 2004, ApJ, 608, 742

Ferguson, H. C., \& Binggeli, B. 1994, A\&ARv, 6, 67

Fontana, A., Pozzetti, L., Donnarumma, I., et al. 2004, A\&A, 424, 23

Frei, Z., Guhathakurta, P., Gunn, J. E., \& Tyson, J. A. 1996, AJ, 111, 174

Fukugita, M., Takahara, F., Yamashita, K., \& Yoshii, Y. 1990, ApJ, 361, L1

Gardner, J. P., Cowie, L. L., \& Wainscoat, R. J. 1993, ApJ, 415, L9

Gardner, J. P., Sharples, R. M., Carrasco, B. E., \& Frenk, C. S. 1996, MNRAS, 282, L1

Glassman, T. M., Larkin, J. E., \& Lafreniere, D. 2002, ApJ, 581, 865

Huang, J.-S., Cowie, L. L., Gardner, J. P., et al. 1997, ApJ, 476, 12

Huang, J.-S., Thompson, D., Kümmel, M. W., et al. 2001, A\&A, 368, 787

Hutchings, J. B., Stetson, P. B., Robin, A., \& Webb, T. 2002, PASP, 114, 761

Ilbert, O., et al. 2006, [arXiv: astro-ph/0604010]

Impey, C. D., Sprayberry, D., Irwin, M. J., \& Bothun, G. D. 1996, ApJS, 105, 209

Jimenez, R., \& Kashlinsky, A. 1999, ApJ, 511, 16

Kauffmann, G., White, S. D. M., \& Guiderdoni, B. 1993, MNRAS, 264, 201

Labbé, I., Rudnick, G., Franx, M., et al. 2003, ApJ, 591, L95

Lambas, D. G., Maddox, S. J., \& Loveday, J. 1992, MNRAS, 258, 404

Larkin, J. E., \& Glassman, T. M. 1999, PASP, 111, 1410

Larkin, J. E., Glassman, T. M., Wizinowich, P., et al. 2000, PASP, 112, 1526

Lenzen, R., Hofmann, R., Bizenberger, P., \& Tusche, A. 1998, Proc. SPIE, 3354, 606

Madau, P., Ferguson, H. C., Dickinson, M. E., et al. 1996, MNRAS, 283, 1388

Maihara, T., Iwamuro, F., Tanabe, H., et al. 2001, PASJ, 53, 25

Mannucci, F., Basile, F., Poggianti, B. M., et al. 2001, MNRAS, 326, 745

Mannucci, F., Della Valle, M., Panagia, N., et al. 2005 A\&A, 433, 807

Martini, P. 2001, AJ, 121, 2301

McIntosh, D. H., Bell, E. F., Rix, H.-W., et al. 2005, ApJ, 632, 191

McLeod, B. A., Bernstein, G. M., Rieke, M. J., Tollestrup, E. V., \& Fazio, G. G. 1995, ApJS, 96, 117

Melbourne, J., Wright, S. A., Barczys, M., et al. 2005, ApJ, 625, L27

Minezaki, T., Kobayashi, Y., Yoshii, Y., \& Peterson, B. A. 1998, ApJ, 494, 11
Minowa, Y., Kobayashi, N., Yoshii, Y., et al. 2005, ApJ, 629, 29

Mo, H. J., Mao, S., \& White, S. D. M. 1998, MNRAS, 295, 319

Moustakas, L. A., Davis, M., Graham, J. R., et al. 1997, ApJ, 475, 445

Nagashima, M., Yoshii, Y., Totani, T., \& Gouda, N. 2002, ApJ, 578, 675

Nagashima, M., Yahagi, H., Enoki, M., Yoshii, Y., \& Gouda, N. 2005, ApJ, 634, 26

Pannella, M., Hopp, U., Saglia, R. P., et al. 2006, ApJ, 639, L1

Papovich, C., Dickinson, M., Giavalisco, M., Conselice, C. J., \& Ferguson, H. C. 2005, ApJ, 631, 101

Peng, C. Y., Ho, L. C., Impey, C. D., \& Rix, H. 2002, AJ, 124, 266

Persson, S. E., Murphy, D. C., Krzeminski, W., Roth, M., \& Rieke, M. J. 1998, AJ, 116, 2475

Pozzetti, L., Bruzual, G. A., \& Zamorani, G. 1996, MNRAS, 281, 953

Pozzetti, L., Madau, P., Zamorani, G., Ferguson, H. C., \& Bruzual, C. A. 1998, MNRAS, 298, 1133

Ravindranath, S., Ferguson, H. C., Conselice, C., et al. 2004, ApJ, 604, L9

Rocca-Volmerange, B., \& Guiderdoni, B. 1990, MNRAS, 247, 166

Rousset, G., Lacombe, F., Puget, P., et al. 2003, Proc. SPIE, 4839, 140

Saracco, P., D’Odorico, S., Moorwood, A., et al. 1999, A\&A, 349, 751

Saracco, P., Giallongo, E., Cristiani, S., et al. 2001, A\&A, 375, 1

Sérsic, J. L. 1968, Atlas de galaxias australes (Cordoba, Argentina: Observatorio Astronomico)

Simard, L., Koo, D. C., Faber, S. M., et al. 1999, ApJ, 519, 563

Somerville, R. S., \& Primack, J. R. 1999, MNRAS, 310, 1087

Somerville, R. S., et al. 2004, ApJ, 600, L135

Steidel, C. C., Adelberger, K. L., Giavalisco, M., Dickinson, M., \& Pettini, M. 1999, ApJ, 519, 1

Steinbring, E., Metevier, A. J., Norton, S. A., et al. 2004, ApJS, 155, 15

Teplitz, H. I., Gardner, J. P., Malumuth, E. M., \& Heap, S. 1998, ApJ, 507, L17

Tinsley, B. M. 1980, Fund. Cosmic Phys., 5, 287

Totani, T., \& Yoshii, Y. 2000, ApJ, 540, 81

Totani, T., Yoshii, Y., Maihara, T., Iwamuro, F., \& Motohara, K. 2001, ApJ, 559, 592

Totani, T., Yoshii, Y., Iwamuro, F., Maihara, T., \& Motohara, K. 2001, ApJ, 558, L87

Trujillo, I., \& Aguerri, J. A. L. 2004, MNRAS, 355, 82

Trujillo, I., Forster Schreiber, N., Rudnick, G., et al. 2005 [arXiv:astro-ph/0504225]

Väisänen, P., Tollestrup, E. V., Willner, S. P., \& Cohen, M. 2000, ApJ, 540, 593

Yan, L., McCarthy, P. J., Storrie-Lombardi, L. J., \& Weymann, R. J. 1998, ApJ, 503, L19

Yoshii, Y., \& Takahara, F. 1988, ApJ, 326, 1

Yoshii, Y., \& Peterson, B. A. 1995, ApJ, 444, 15 